\title{
H2BC13 Gene
}

National Cancer Institute

\section{Source}

National Cancer Institute. H2BC13 Gene. NCI Thesaurus. Code C162950.

This gene is involved in chromatin packaging. 